\title{
Design of Remote Fitting Platform Based on Virtual Clothing Sales
}

Miao Yu, Shanghai Business School, China

\begin{abstract}
To improve the convenience and effect of fitting process, an online fitting system of user-personalized body type based on the virtual technology is put forward. Firstly, it develops the fitting system with Java as the development language and constructs the human model, spinning motion model, and virtual clothing model; then, it divides the relevant model creation based on the body, generates the personalized body type, and designs the slab model, represented by triangular patch, while all points in same gender model keep the spatial position relation with each other to simplify the interpolation process; finally, it verifies the efficiency of the developed system through the factor analysis and cluster fitting design.
\end{abstract}

\section{KEYWORDS}

Body Division, Fitting System, Virtual Clothing, Virtual Technology

\section{INTRODUCTION}

In recent years, with the continuous development of computer software level, the continuous improvement of hardware performance and the continuous popularity of 3D scanning equipment, the threshold of 3D data acquisition has been greatly reduced. Nowadays, 3D data has become another important digital media following sound, image and video. 3D human body and virtual clothing, as two common 3D models, have always been an important research content in computer graphics and machine vision, and have been widely used in film and television, games, clothing design, virtual fitting, e-commerce and many other areas of people's livelihood.

The virtual clothing is one of the research hotspots in the virtual reality and computer graphics field. As the foreign clothing e-commerce has developed very early, most clothing websites have already can provide the 3D view, purchase history record, virtual fitting room, auxiliary facilities and other services. For example, the famous fitting website - My Virtual Model provided the online fitting service of human body measurement; the scientists from the Germany Fraunhofer Gesellschaft and other scientific research team jointly develop a set of fitting system. Virtual clothing, is a digital fashion using computer technology to simulate the fabric. The comprehensive consideration of clothing sample version, fabric characteristics, body and human movements, and clothing shape and its changes, are all obtained through computer simulation. 
Virtual clothing display is the use of holographic technology, the Internet or digital interactive large screen to show the dynamic visual effects when the human body or the digital model-"dressing" virtual clothing. With the development of digital media art, interactive and three-dimensional display technology, virtual clothing display system is widely used in 3-D fashion design, fashion shows, entertainment, "virtual dressing system" in shopping malls and television advertising area. This paper analyzes and compares several technologies in recent years, summarizing a human computer interaction model. It is concluded that, with the development of smart environment technologies such as holographic, somatosensory interactive, three-dimensional vision and augmented reality, a personalized, stylish and intelligent virtual fitting experience as the representative of "private order" will be an important part.

When debugging large applications, computers have a low configuration. Visual $\mathrm{C}++$ provides remote debugging capability to facilitate program developers to debug programs stored on highly configured machines.

In the garment industry, the application of three-dimensional human body/garment modeling and simulation is booming, and personalized customization is gradually becoming the norm. The continuous improvement of the accuracy of 3D human body reconstruction will certainly bring new opportunities for the personalized advanced customization industry. Traditional clothing design requires designers to have a variety of professional skills such as plate making, code placing, sewing, etc. Most importantly, in the design process, it is impossible to directly see the fitting effect of clothing, but can only operate the two-dimensional cut pieces. In contrast, mature 3D clothing simulation software such as Optitex3D in the US, Lecra in France, AGMS Industry3D in Japan, Clo3D and Marvelous Designer in Korea, and 3D modeling software such as Maya, Blender et al. can directly sew a 2D garment cut piece into a 3D garment and wear it on a 3D human body. By using the physical simulation technology of fabric, the drape, fold and other real effects of the garment on the human body can be generated.

Comparing to the foreign fitting system, the relevant domestic research also can get a certain progress. The recent representative researches include: Literature (Li Y, Zhang W, Fan J.(2014)) introduces the development history and current condition of virtual clothing, and pays attention to introduce the research works of various research institutes; Literature (Luximon A.(2015)) gives the improved realistic simulation equation of material point-spring model and clothing by analyzing the basic steps of clothing virtual simulation, and carry out the solution of dynamic system; Literature (Miao L, Shang S Y, Cai C X.(2013)) researches the 3D clothing simulation technique in the virtual human animation, including the virtual human body modeling, character animation, physical simulation of cloth and collision detection and response of deformable body, of which the overall objective is to construct a complete $3 \mathrm{D}$ clothing simulation environment and complete the development of efficient algorithm around the system real-time performance.

Throughout the research status of virtual clothing, it mainly centralizes in four aspects: a) parametric human body figure modeling. The efficient reconstruction and generation of high-precision 3D human body. The clothes involved in the virtual fitting should be fit first, followed by visual beauty. The fit is the foundation, and the visual effect is to further enhance the user experience. And the combination must have a high precision three-dimensional human body as the fundamental. If put the cart before the horse, excessive gorgeous visual effect will only mislead consumers, not conducive to reducing the rate of return of clothing products; b) dynamic display of 3D clothing provides the character animation support and the rapid generation of realistic clothing; c) calculation efficiency and realistic of physical modeling of cloth; d) real-time interaction. As the physical modeling of cloth has huge calculated amount, how to carry out the high-quality simulation at the low-end hardware platform or interactive environment (such as the normal PC or video game) is a new challenge (Zhu X J, Xiong X Y. (2016)). This paper introduces the idea of artificial life into the clothing texture generation, develops the intelligent simulation system of 3D virtual fitting model on the basis of mixed virtual reality technology, construct the 3D virtual model similar to the actual human body in the 3D 
space as the fitting substitute of consumer, design and realize the scene screen function at the same time, which enables the consumers to more visually and freely observe the dressing effect through the virtual platform; e) to improve the utilization rate of existing three-dimensional garments, so that they can be applied to a variety of body types quickly and efficiently and maintain the sense of reality of garments, namely garment retargeting or garment Nowadays, three-dimensional human body and three-dimensional clothing are still in the rise in the field of fitting, and their related technologies still have great room for improvement and research value, which are worthy of further exploration and exploration. This is also the research goal and focus of this paper.

\section{ONLINE FITTING SYSTEM}

The online fitting system applies Java as the development language to be applied into the network, and the whole system is designed as an Applet, to be expediently embedded into Html page. The user open and operate the fitting system by IE browser. Our system realizes the selection, fitting, display and other functions necessary to the virtual fitting room.

With the development of retail industry, the online clothing industry needs a large number of realistic virtual clothing models. Therefore, in the virtual clothing fitting environment, there are a variety of studies on the tailoring and interaction of clothing to achieve the generation of virtual clothing. The common virtual costume design process includes costume pattern modification and costume making procedure. First of all, present the designed 2D clothing pattern and modify the model pattern to create a new model style line, including sleeve stretching, internal cutting, etc. Then, the modified 2D pattern is stitched around the mannequin to form a virtual dangling 3D garment. These studies either focus on fabric simulation without paying attention to fit evaluation, or only focus on fit evaluation without paying attention to interaction design of clothing patterns. In order to design various styles of clothing and achieve satisfactory results, we focus on a novel virtual fitting solution that includes fitting evaluation and pattern modification to design various types of clothing and speed up the virtual clothing design process.

\section{Virtual Model}

Java technology is applied in the design of online fitting system. To be convenient for the model to display in Applet, and to load as fast as possible when starting the system, we choose the obj format in several model document formats supported by Java. The system provides multiple virtual models of different figures. After users make the choice at the model list, the system will display the virtual model and enable it to make the spinning around the $\mathrm{Y}$ axis (as shown in Figure 1) to be convenient for the user to view whether the figure of virtual model is matched with one's own figure. The autorotation of virtual model around the $\mathrm{Y}$ axis is achieved by using the Alpha object and interpolator.

\section{Virtual Clothing}

As the online fitting system uses the 2D clipping data in the clothing model document to construct the 3D virtual clothing, while most of the edges between the cloth strip and cloth strip cannot completely match each other, we give the seamless splice algorithm of cloth strip for sewing these cloth strips. Taking sewing two cloth strips at front and rear of the main body (not including the shoulder girdle) of a waistcoat as the example, we shall set forth how the seamless splice algorithm works. Firstly, the system gets the vertex set of cloth strip in the clothing model document. At the time, the cloth strip is flat, and all $\mathrm{Z}$ components of point coordinate in grid are 0 . The system uses some conics to simulate the latitude line on the cloth strip, and assigns the $\mathrm{Z}$ component of vertex coordinate on latitude line according to the conic equation. The conic applied in our test is $\mathrm{X} 2 / 16+\mathrm{Z} 2 / 9=\mathrm{C}(\mathrm{Z}>0$ is taken for the front strip, while $\mathrm{Z}<0$ is taken for the rear strip). For the virtual model given in the Figure 1, we take 0.02 as the value of $C$, as this value can roll the cloth strip in cylindroids relatively closely to surround the model. After the cloth strip gets the curvature 

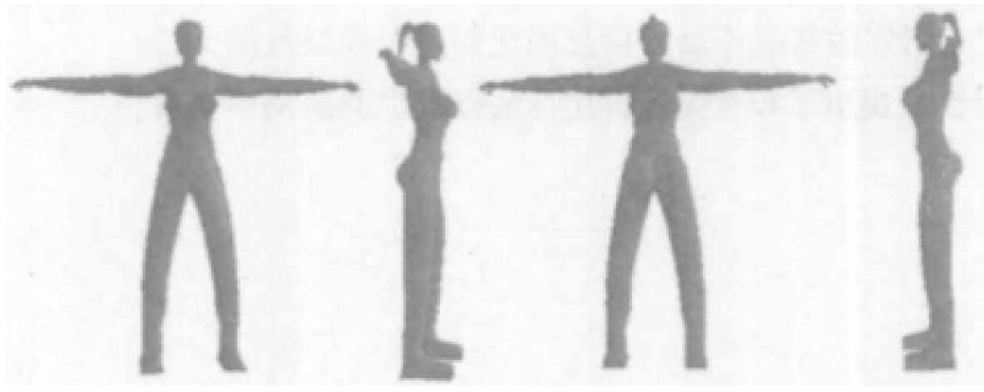

and is vertically put around the virtual model, we use the scanning plane to assist the splice. The scanning plane is a family of horizontal plane, which passes through each cloth strip from the bottom to the top. It is a process for the $\mathrm{Y}$ coordinate component to increase, while the step length of increase is depended on the accuracy that the triangulation shall achieve. In our test, the 001 order is taken as the step length. For the sewing line parallel to the $\mathrm{Y}$ axis, the scanning plane is parallel to $\mathrm{X} \_\mathrm{Z}$ plane, while the sewing lines of other directions can be solved by rotating the coordinate system. The scanning lines can be obtained by intersecting the cloth strip and scanning plane, which actually are the latitude lines of cloth strip, and their projection on the $\mathrm{X}$ _Y plane is the straight line, and that on X_Z plane is the conic. Sample the endpoint of scanning line once a certain interval, take the endpoint of scanning line at the side adjacent to two cloth strips as the vertex set, and make the triangulation. Therefore, two cloth strips are linked together. The Figure 2 is the simple schematic diagram for splicing the cloth strips.

\section{FITTING PROCESS AND METHOD FOR REALIZING THE USER-PERSONALIZED BODY TYPE}

\section{Personalized Formulation Step}

Combing with advantages of two technologies and effectively mixing these two technologies, this paper forms the fitting method based on body type formulated with user personalization to meet the requirements of different users toward the personalized fitting. The realization step of method is as follows, shown in Figure 3:

Figure 2. Schematic diagram for seamless splice algorithm of cloth strip
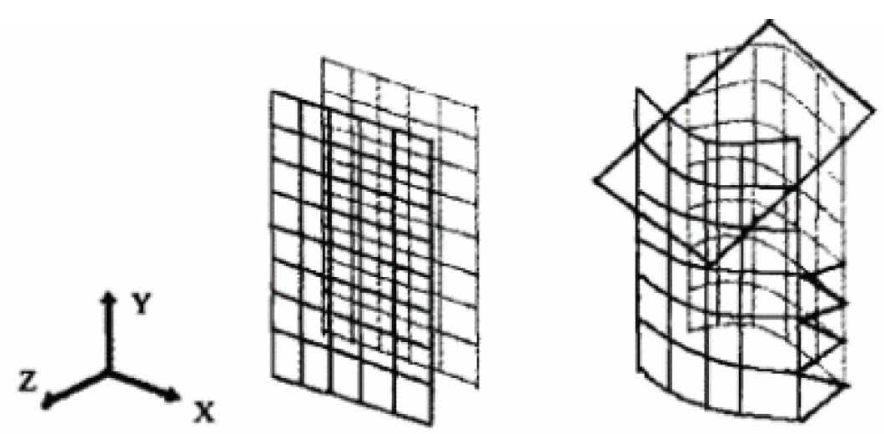


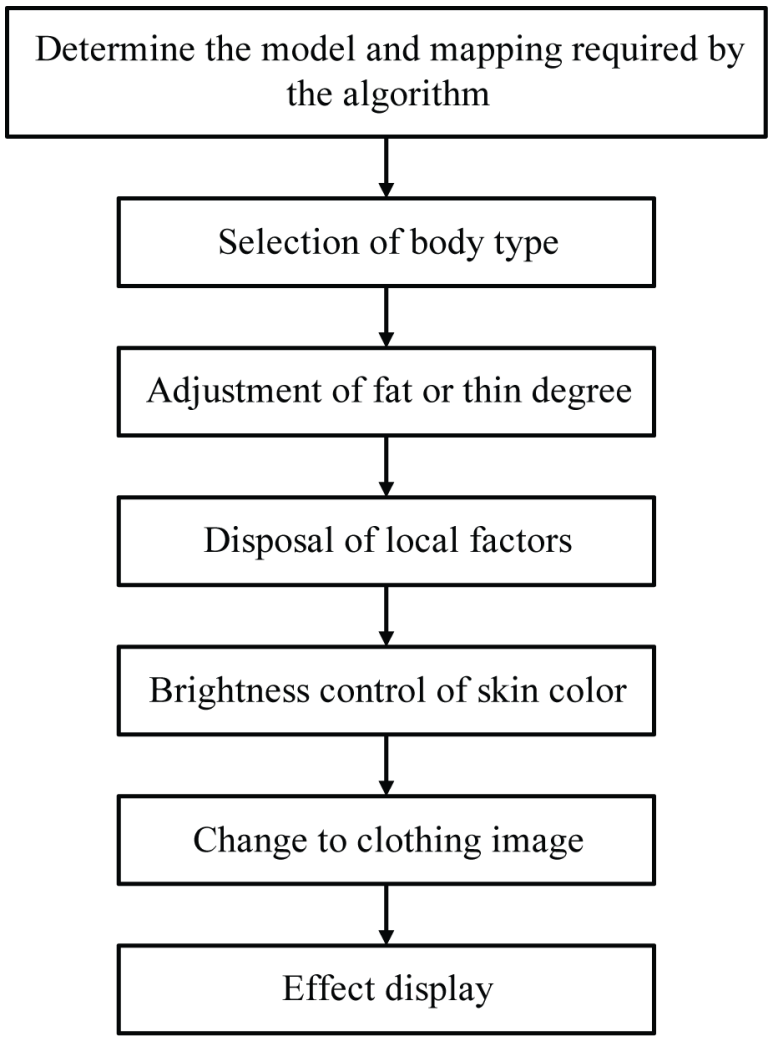

1. The user sets his/her gender to be convenient for the system to distinguish the required 3D model and mapping.

2. Choose the standard body type suitable to the user figure (Type "S", "H", "A" and "Y").

3. The user adjusts the fat or thin degree of body type according to the demand, to generate the user-personalized body model.

4. Aiming at the difference between the female chest and male abdomen, the user can partially make the special adjustment.

5. Carry out the shape change to the clothing image matching to the original model, enable the clothing mapping to match with the final body image, and complete the fitting process.

\section{Body Division and Establishment of Relevant Models}

To clearly present the body type of user, the classification to the body type of Chinese male and female must be carried out. According to the existing classification in connection to the body type, the female body type is usually divided into four kinds of standard types: Type " $\mathrm{S}$ " is that the chest and buttock are plump, while the waist is relatively slender; Type " $\mathrm{H}$ " is that the chest, waist and buttock are relatively uniform; Type " $\mathrm{A}$ " is that the chest is relatively small, while the waist and buttock are relatively obvious; Type "Y" is that the chest is plump, while the waist and buttock are uniform. Use the 3D processing software to create the relevant models. In the experiment, use the Maya modeling software, create a slab model corresponding to the original model, and define it as $M_{0}$ (two different models distinguished according to the gender, similarly hereinafter). When creating the slab model, in addition to the body, it is considered that there may be the clothing area, while such areas have been created for multiple 
layers - clothing area, of which these areas are created by outward diffusing according to the model body profile - the closer to the body profile it is, the intensive the line is. In this paper, three layers of lines are additionally covered on the clothing areas possibly to be at the upper half part of model body, while six layers of lines are additionally covered on the bottom half part of body as the skirt in the female clothing is considered. Meanwhile, more detailed lines are made to the chest of female model and abdomen of male model, as shown in Figure 4.

According to the features of four determined body types, modify the position of all coordinates of model. Such adjustment shall satisfy the fat accumulation process of human body (12), successively create four models of " $M S$ ", " $M \mathrm{H}$ ", " $M \mathrm{~A}$ " and " $M \mathrm{Y}$ ", respectively corresponding to these four types. However, considered that the figure of most users would not completely match to one of these four standard types, and there still is the fat or thin difference among the crowd with same one body type, the thin ("min") and fat ("max") models are respectively made for each kinds of body type, so that, there are 12 models of "Smax", "MS", "Smin", " $Y \max$ ", "MY", " $Y \min "$, "Hmax", "MH", "Hmin" and so on, while these models also are the models used for the shape changes of clothing, with effect as shown in Figure 5.

During the modification process for point position of model, in addition to considering the changes to the body type, the clothing area attached to around the body profile shall be considered. As the shape change to the body shape will influence the clothing area, namely, during the model adjustment process, following the change of body profile, the clothing area shall be made relevant

Figure 4. Slab model of original model
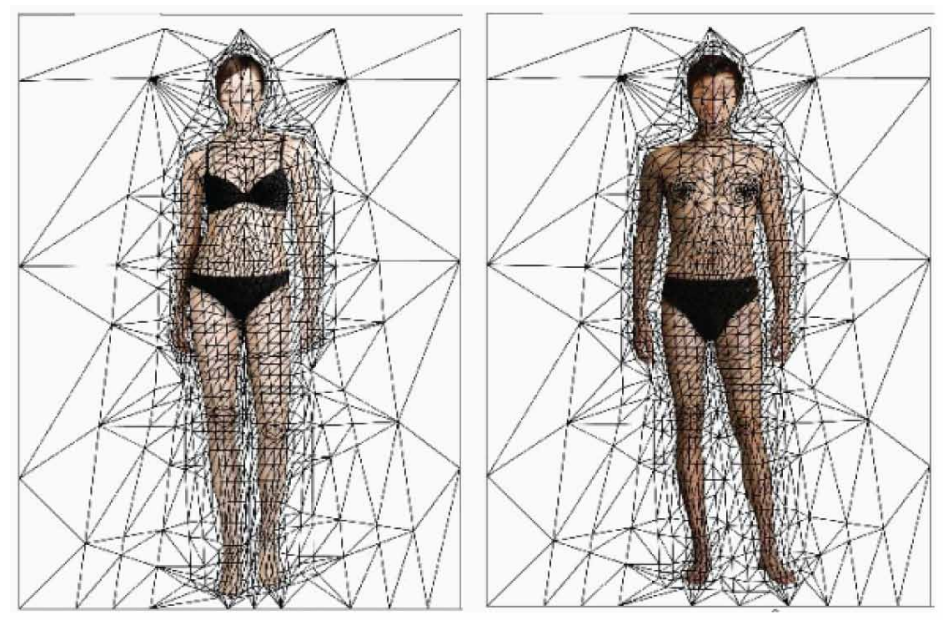

Figure 5. Body type changes of three kinds of models and corresponding models

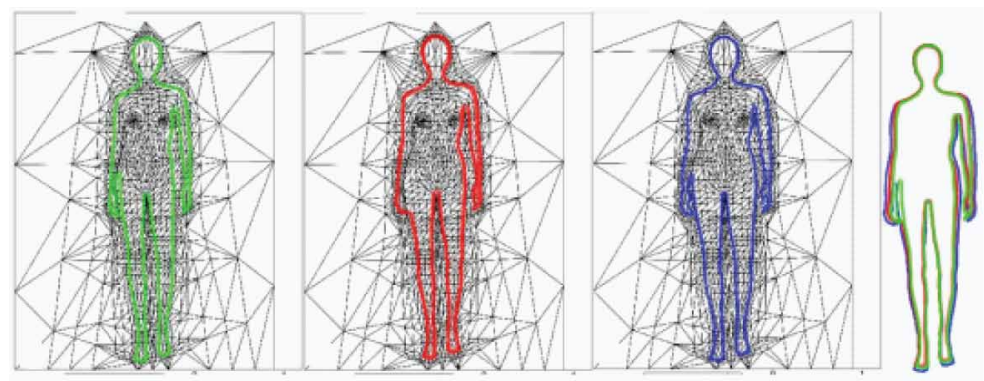


associate adjustment - such association is stronger in the area closer to the body profile. This paper modifies the model according to the clothing with maximum peripheral profile in the normal clothing, for example, the females wear the down jacket and skirt, while males dress on the dust coat and pants. The model creation of male is similar to that of female, with four types of " $\mathrm{S}$ ", " $\mathrm{H}$ ", "A" and " $\mathrm{Y}$ " and corresponding "fat and thin" versions of each type for type division.

\section{Generation Process of Personalized Body Type}

The slab model is represented finally by the triangular patch, while all points in the model of same one gender keep the spatial position relation among each other, which can simplify the interpolation process. The interpolation process is as follows:

1. The interpolation from the normal model to one of four standard figures: Considered that the figure of original model may not belong to these four standard body types, the interpolation process is required; if the original model belongs to one defined standard model, then this step can be skipped.

2. Interpolation suitable to the fat or thin requirement: The figure of user cannot be generalized only by the standard body type, while the fat and thin difference shall be considered. From the "standard figure" to the "fat" or the "thin" are two interpolation processes of different directions and the difference of such variation trend must be considered in the algorithm.

3. Processing of partial body factors: The partial factor is for the female chest and male abdomen and body muscle, while such partial processing can satisfy the personalized demand of user more.

4. Processing of skin color: Change the skin color of mapping, to fit the skin color value as required by the user.

Therefore, get the target point set, mapping point set and point sequence information necessary in the 3D technology, and finally obtain the 3D model defined according to the feature of userpersonalized body type.

\section{OBJECTIVE EVALUATION OF MODELING STYLE INDEX}

\section{Factor Analysis}

To reflect the relation among 15 selected modeling style indexes and modeling, this paper applies the factor analysis to carry out the principal factor analysis for the modeling style index data of 55 skirt samples, namely, carry out the factor analysis for the data in Table 2. Integrate 15 indexes as several principal factors and represent the relation between each index and principal factor. Through the factor analysis, it can be found that which indexes have great influence to the modeling, to provide the foundation for the further analysis of relation between the fabric performance and modeling. Firstly, carry out the standardization processing to the original data in the Table 2, with the extraction method of factor as the main componential analysis, and make the rotation of maximum variance for the principal factor result (rotation method with maximum variance of Kaiser standardization is applied), with its result as shown in Table 1. From the Table 1, it can be seen that, 4 principal factors can be extracted, with accumulative contribution rate of each principal factor up to $83.291 \%$ before rotating.

According to the representation meaning of original variables, the explanation and definition can be made to 4 principal factors.

Factor 1: Profile factor, mainly constituted by the side clothing angle of spread, front clothing angle of spread, included angle of mean wave crest, wave number, central front length of skirt, included angle of mean wave trough, front clothing width and standard deviation of wave trough diameter. These indexes mainly reflect the overall modeling profile of clothing. The accumulative 
Table 1. Contribution rate of principal factor

\begin{tabular}{|c|c|c|c|c|c|c|}
\hline \multirow{2}{*}{$\begin{array}{l}\text { Principal } \\
\text { factor }\end{array}$} & \multicolumn{3}{|c|}{ Contribution rate of principal factor } & \multicolumn{3}{|c|}{$\begin{array}{c}\text { Contribution rate of principal factor after } \\
\text { rotation }\end{array}$} \\
\hline & $\begin{array}{l}\text { Total } \\
\text { value }\end{array}$ & $\begin{array}{l}\text { Contribution } \\
\text { rate/\% }\end{array}$ & $\begin{array}{c}\text { Accumulative } \\
\text { contribution } \\
\text { rate/ } \%\end{array}$ & $\begin{array}{l}\text { Total } \\
\text { value }\end{array}$ & $\begin{array}{l}\text { Contribution } \\
\text { rate/ } \%\end{array}$ & $\begin{array}{c}\text { Accumulative } \\
\text { contribution } \\
\text { rate } / \%\end{array}$ \\
\hline 1 & 6.630 & 44.198 & 44.198 & 5.998 & 39.990 & 39.990 \\
\hline 2 & 3.071 & 20.458 & 64.668 & 2.943 & 19.626 & 59.628 \\
\hline 3 & 1.572 & 10.473 & 75.143 & 2.118 & 14.127 & 73.745 \\
\hline 4 & 1.221 & 8.146 & 83.287 & 1.425 & 9.543 & 83.289 \\
\hline
\end{tabular}

contribution rate of square load for extracted value of the profile factor reaches $39.991 \%$, which is the main factor influencing the modeling.

Factor 2: Line factor of wave crest, mainly constituted by the mean wave crest diameter, standard deviation of wave crest diameter and area of the bottom. These features mainly reflect the wave crest depth of clothing and variation degree of wave depth, and the accumulative contribution rate of square load for extracted value of the factor reaches $19.627 \%$.

Factor 3: Line factor of wave trough, mainly constituted by the mean wave trough diameter, area of the bottom, included angle of mean wave crest, wave number and included angle of mean wave crest. These features mainly reflects the wave trough depth of clothing and variation degree of wave trough, and the accumulative rate of square load for extracted value of the factor reaches $14.128 \%$.

Factor 4: Detail factor, mainly constituted by the standard deviation for included angle of wave crest and wave trough, and reflecting the rhythm and uniform sensation of clothing wave fold. The accumulative contribution rate of square load for extracted value of detail factor reaches $9.544 \%$.

\section{Design Results}

With 15 modeling evaluation index data of 55 clothing samples as the basis, carry out the cluster design. The divided object is 55 skirt samples, namely, the domain of discourse $U=\left\{f_{1}, f_{2}, f_{3}, \ldots, f_{55}\right\}$, representing the character of each object with 15 indexes, namely, $X=\left\{X_{1}, X_{2}, \ldots, X_{15}\right\}$ as the index set, and the original data matrix is obtained. After standardizing and normalizing the original data matrix, carry out the cluster for it by utilizing the cluster method, with the design result of cluster fitting as shown in Figure 6.

Compared to the past plane fitting technology (not make the personalized adjustment processing to the body type, while the fitting process is only to simply cover the clothing mapping on the model photo), the overall time consumed for executing the cloth changing order every time is about $484 \mathrm{~ms}$ (data acquisition platform and loaded image material is in correspondence to this experiment). In comparison, the algorithm adds the additional calculation burden. However, after determining the body type, the additional time consumed will obviously decrease, and the algorithm can reflect the personalized fitting demand, with ideal display effect. The Figure 6 is the contrast display of dressing effect, it can be seen that, after the dressing design of virtual platform in this paper, the individual image of model promotes.

\section{CONCLUSION}

With the development of computer technology and network technology, more and more consumers buy daily consumer goods such as clothing and accessories through e-commerce websites such as 


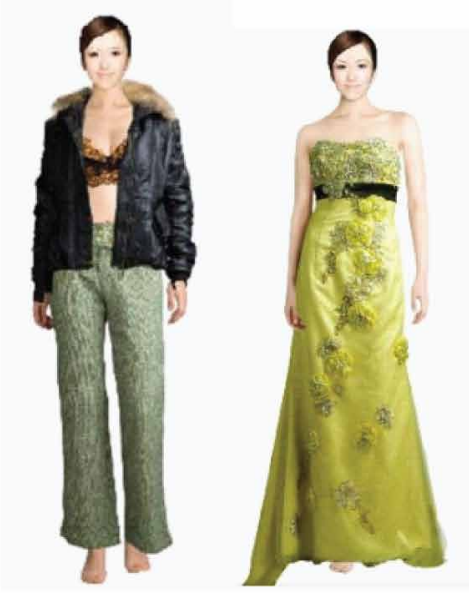

(a) Result of female dressing experiment (the left side is the original clothing)

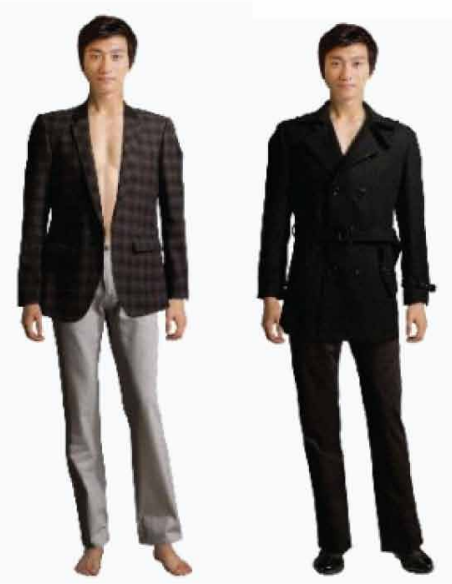

(b) Result of male dressing experiment (the left side is the original clothing)

Taobao. But buying clothes online is different from shopping malls in daily life, online shopping clothes fit, whether the color and style are suitable for themselves, consumers can not try on before buying. Therefore, when buying clothes online, if there is a "virtual" model to help consumers try on, let consumers "try on" their favorite clothes before paying, so that users can go to the clothing mall, it is an exciting thing for consumers. The birth of digital human modeling technology not only solves the problem of the selection details of the online clothing purchase, but also will make the online shopping services more humanized.

The situation of the three-dimensional clothing CAD at present is introduced firstly, then some key technologies are emphasized, such as the three-dimensional body measurement technology, the clothing human body modelling, the virtual clothing display as well as the clothing 3D/2D transformation.

Digital virtual technology is fully and materially penetrated into the traditional clothing design and manufacturing industry. Together with material science, before the real fabric cutting link occurs, these virtual technologies can better predict the future potential problems in advance, provide better solutions, and then start to implement the subsequent production links. This may be three to five times more pragmatic than the fashion environmental slogan is, which will save considerable costs 
and resources for the industry every year. It's possible to redefine the rules of the show and getting more computer $3 \mathrm{D}$ models.

At present people research on virtual fitting technology under the network environment Mostly concentrated on the 3D virtual fitting, i.e., with 3D body scanning first get consumers shape data, and based on 3D human body modeling technology is used to establish consumer 3D human body model, and then the "wear" three-dimensional simulation of clothing to the virtual model. Virtual fitting technology is still in the research process, and there is still a long way to go before it can be successfully applied to online clothing sales. The few virtual fitting software that has appeared in the market are also at the stage of exploration and trial. The fitting effect is not ideal, and the most prominent problem is that the sense of reality is far from enough. In the process of constructing the virtual models of consumer, the consumer of the surface characteristics are not under enough attention, which is the important factors that affect consumers cognitive psychology, because the current use of computer simulation of consumer's facial features are still not mature, the resulting network model and the consumer is far my facial features. In this case, it will be easier for consumers to accept facial features with the combination of two-dimensional image technology. With the rapid development of e-commerce, network fitting technology is bound to enter the field of clothing industry. It will greatly improve the efficiency of online clothing sales. With the development of the Internet and the progress of virtual clothing and virtual human technology, the success of online fitting technology is in sight.

The research develops a kind of online fitting system of user-personalized body shape based on virtual technology, construct the human body model, spinning motion model and virtual clothing model, representing by triangular patch, while all points in the model of same one gender keep the spatial position relation among each other, to simplify the interpolation process. The experiment result verifies the system efficiency. The key points of research in next step are: a) In the creation of virtual model, if wanting to enable the user to get more real fitting experience, the body motion capture system can be employed to enable the model to represent various chronic motion of "master" in the real life, to make the clothing dressing to be more personalized. B) In the intelligent evaluation, in addition to giving out the evaluation on clothing matching fitness, with combination of emotion model and other technologies, the system can automatically predict the favor pattern of the user and give the recommended clothing matching suggestion. 


\section{REFERENCES}

Chen, Y., Cheng, Z.-Q., \& Lai, C. (2015). Realtime reconstruction of an animating human body from a single depth camera. IEEE Transactions on Visualization and Computer Graphics, 22(8), 2000-2011.

Floater, M. S. (2015). Generalized barycentric coordinates and applications. Acta Numerica, 24(1), $161-214$.

Gordo, A., Almazan, J., \& Revaud, J. (2016). Deep Image Retrieval: Learning Global Representations for Image Search. European Conference on Computer Vision, 241-257.

Kunitomo, S., Nakamura, S., \& Morishima, S. (2010). Optimization of Cloth Simulation Parameters by Considering Static and Dynamic Features. ACM SIGGRAPH Posters, 15, 1-2.

Li, B., \& Univ, B. H. (2016). Robust aircraft segmentation from very high-resolution images based on bottom-up and top-down cue integration. Journal of Applied Remote Sensing, 10(1), 975-979.

Li, Y., Zhang, W., \& Fan, J. (2014). Study on clothing pressure distribution of calf based on finite element method. Journal of the Textile Institute, 105(9), 955-961.

Lin, Y. L., \& Wang, M. J. J. (2016). The Development of a Clothing Fit Evaluation System Under Virtual Environment. Multimedia Tools and Applications, 75(13), 7575-7587.

Loper, M., Mahmood, N., \& Romero, J. (2015). SMPL: A skinned multi-person linear model(J). ACM Transactions on Graphics, 34(6), 248.

Luximon, A. (2015). Functional 3D Human Model Design: A Pilot Study Based on Surface Anthropometry and Infrared Thermography. Computer-Aided Design and Applications, 12(4), 475-484.

Maclean, K. E., Smith, J., \& Pasquero, J. (2015). Building a Haptic Language: Communication through Touch. Cs.ubc.ca, 38(7), 1434-1452.

Miao, L., Shang, S. Y., \& Cai, C. X. (2013). Research on Image Binding Mechanism Based on Kinect Skeletal Tracking in Virtual Fitting System. Applied Mechanics and Materials, 376, 437-440.

Perronnin, F., Sanchez, J., \& Mensink, T. (2010). Improving the Fisher Kernel for Large-Scale Irnage Classification. European Conference on Computer Vision, 115(7), 143-156.

Ren, Z., \& Huang, Z. (2015). Determination of glucose concentration based on pulsed laser induced photoacoustic technique and least square fitting algorithm. Proceedings of SPIE - The International Society for Optical Engineering, 9619.

Sabina, O., Elena, S., Emilia, F., \& Adrian, S. (2014). Virtual Fitting - Innovative Technology for Customize Clothing Design. Procedia Engineering, 69(1), 555-564. doi:10.1016/j.proeng.2014.03.026

Song, D., Tong, R., \& Chang, J. (2016). 3D body shapes estimation from dressed human silhouettes. Computer Graphics Forum, 35(7), 147-156.

Tao, X., \& Bruniaux, P. (2013). Toward advanced three-dimensional modeling of garment prototype from draping technique. International Journal of Clothing Science and Technology, 25(4), 266-283.

Wang, H. M. (2018). Rule-Free Sewing Pattern Adjustment with Precision and Efficiency. ACM Transactions on Graphics, 37(4), 1-13.

Wu. (2014). Study on the Design of Virtual Apparel Fitting System and the Application of Motion Capture Technology in Electronic Commerce. Applied Mechanics and Materials, 519-520, 403-409.

Yu, U. J., \& Damhorst, M. L. (2015). Body Satisfaction as Antecedent to Virtual Product Experience in an Online Apparel Shopping Context. Clothing \& Textiles Research Journal, 33(1), 3-18.

Yu, X. H., \& Pan, L. T. (2013). The Research on Reconstructing the Virtual Environment of E-Commerce Clothing(J). Applied Mechanics and Materials, 380-384, 2251-2255. 
Zhang, M., Lin, L., \& Pan, Z. (2015). Topology-independent 3D garment fitting for virtual clothing. Multimedia Tools and Applications, 74(9), 3137-3153.

Zhao, T., \& Li, S. (2018). 3-D reconstruction of human body shape from a single commodity depth camera. IEEE Transactions on Multimedia, 21(1), 114-123.

Zhu, X. J., \& Xiong, X. Y. (2016). Human body shapes modeling and feature points location based on ASM for virtual fitting. In Sixth International Conference on Information Science and Technology. IEEE. 